\title{
ON NIU'S CONJECTURE FOR TANDEM QUEUES
}

\author{
BETSY S. GREENBERG, ${ }^{*}$ University of Texas, Austin
}

\begin{abstract}
We show that Niu's (1980) conjecture is true for Poisson arrivals and light traffic. We also show that a weak version of the conjecture is true for a special case in heavy traffic.
\end{abstract}

\section{Introduction}

Based on the intuition that the constant server at the first station in a tandem queue tends to make the arrival process to the second station more regular and thus reduces delay at the second station, Niu (1980) has made the following conjecture.

Conjecture. In a $G I / D / 1 \rightarrow G / 1$ system, the stationary expected delay in front of the second server is smaller than it would be if there were no first server at all. In fact, we believe a stronger conjecture should also be true, namely, the larger the constant service time at the first station, the smaller the stationary expected delay in front of the second server will be. Thus, the original conjecture follows by letting the service time at the first station go to zero.

Often it is difficult to calculate the expected delay at the second station in a tandem queueing system. If the conjecture is true, then it would provide a useful bound on the expected delay at the second station. That is, the expected delay at the second station would be less than if the first station were not there.

We provide the following supporting evidence to Niu's conjecture. In Section 2, we show that Niu's strong conjecture is true for Poisson arrivals and light traffic. In Section 3 , we show that the weak version is true for heavy traffic for the special case where the interarrival variance and the variance of the stochastic service times are equal.

\section{Light traffic approximation}

We use the method developed in Wolff (1982) to approximate the expected delay in queue at the second server for single-server tandem queueing systems in light traffic with Poisson arrivals. The light traffic approximation represents the asymptotic behavior as $\rho$, the server utilization, approaches zero. Using this approximation, we prove Niu's conjecture for the case of Poisson arrivals and light traffic.

Theorem 1 . In an $M / D / 1 \rightarrow G / 1$ system, for sufficiently light traffic, the stationary expected delay in front of the second server is smaller than it would be if there were no first server at all. In fact, the improvement is a monotone function of the constant service time at the first station.

Proof. Let system $A$ be a tandem queueing system with Poisson arrivals, constant service, $S_{1}=s$, at the first station and stochastic service, $S_{2}$, at the second station.

Received 20 October 1986; revision received 8 May 1987.

* Postal address: Department of Management Science and Information Systems, The University of Texas at Austin, CBA 5.202, Austin, TX 78712-1175, USA. 
System $B$ is exactly the same except that $S_{1}=s+h$, where $h>0$. Showing that $d_{2}$, the expected delay at the second server, is less in system $B$ than in system $A$, is equivalent to proving the theorem.

We compare the delay at the second station for systems $A$ and $B$ :

$$
d_{2}^{A}=\lambda s E\left[S_{2}-s\right]^{+}+\lambda E\left[S_{2}\right] E\left[S_{2}^{e}-s\right]^{+}+o\left(\rho^{2}\right),
$$

and

$$
d_{2}^{B}=\lambda(s+h) E\left[S_{2}-s-h\right]^{+}+\lambda E\left[S_{2}\right] E\left[S_{2}^{e}-s-h\right]^{+}+o\left(\rho^{2}\right) .
$$

Now,

$$
\begin{aligned}
E\left[S_{2}-s\right]^{+}= & E\left[S_{2}-s \mid s+h \geqq S_{2}>s\right] P\left\{s+h \geqq S_{2}>s\right\} \\
& +E\left[S_{2}-s \mid S_{2}>s+h\right] P\left\{S_{2}>s+h\right\} \\
= & E\left[S_{2}-s \mid s+h \geqq S_{2}>s\right] P\left\{s+h \geqq S_{2}>s\right\} \\
& +E\left[S_{2}-s-h\right]^{+}+h P\left\{S_{2}>s+h\right\} .
\end{aligned}
$$

We can obtain a similar expression for $E\left[S_{2}^{e}-s\right]^{+}$, so

$$
\begin{aligned}
d_{2}^{A}= & d_{2}^{B}-\lambda h E\left[S_{2}-s-h\right]^{+} \lambda s E\left[S_{2}-s \mid s+h \geqq S_{2}>s\right] P\left\{s+h \geqq S_{2}>s\right\} \\
& +\lambda E\left[S_{2}\right] E\left[S_{2}^{e}-s \mid s+h \geqq S_{2}^{e}>s\right] P\left\{s+h \geqq S_{2}^{e}>s\right\} \\
& +\lambda s h P\left\{S_{2}>s+h\right\}+\lambda E\left[S_{2}\right] h P\left\{S_{2}^{e}>s+h\right\}+o\left(\rho^{2}\right) .
\end{aligned}
$$

Since

$$
E\left[S_{2}\right] P\left\{S_{2}^{e}>s+h\right\}=\int_{s+h}^{\infty} P\left\{S_{2}>x\right\} d x=E\left[S_{2}-s-h\right]^{+},
$$

we have $d_{2}^{A} \geqq d_{2}^{B}$ and the inequality is strict provided $P\left\{S_{2}>s\right\}>0$.

Wolff also obtains an expression approximating the distribution of delay at the second station. Using that expression, and an argument similar to the one above, we may strengthen the conclusion of Theorem 1 to stochastic ordering of delays for strictly increasing distributions. It may be possible to strengthen this result to general distributions using a continuity argument.

\section{Heavy traffic approximation}

Harrison (1978) develops a diffusion approximation for tandem queues in heavy traffic. He derives differential equations for the density of the waiting time distribution, and solves these equations for the special case when the service times at the first station are deterministic and the variance of the service distribution at the second station is equal to the interarrival variance. We use this explicit solution to approximate the expected delay at the second station, and compare this with the expected delay at the second station if it were alone, using Kingman's (1962) heavy traffic approximation.

From this comparison, which is presented in detail in Greenberg (1986), we have the following result.

Theorem 2. If the service time at the first station is constant and equal to the service time at the second station, and the interarrival variance is equal to the service variance at the second station, then in the limit as server utilization approaches 1 , the presence of the first station reduces the expected delay in queue by one quarter compared to when the first station is not there. 


\section{References}

Greenberg, B. S. (1986) Queueing Systems with Returning Customers and the Order of Tandem Queues. Ph.D. Thesis, University of California, Berkeley.

HARRISON, J. M. (1978) The diffusion approximation for tandem queues in heavy traffic. Adv. Appl. Prob. 10, 886-905.

KInGman, J. F. C. (1962) Some inequalities for the queue $G I / G / 1$. Biometrika 47, 315-324.

Niu, Shun-Chen (1980) Bounds for the expected delays in some tandem queues. J. Appl. Prob. 17, 831-838.

WolfF, R. W. (1982) Tandem queues with dependent service times in light traffic. Operat. Res. 30, 619-635. 